\title{
The overcoming high pre-transplant isoagglutinin titers using intravenous immunoglobulin, booster rituximab, salvage plasmapheresis in ABO-incompatible living donor liver transplantation: a case report
}

\author{
Hyung Hwan Moon, Ji Hoon Jo, Young Il Choi, Dong Hoon Shin
}

Department of Surgery, Kosin University Gospel Hospital, Busan, Korea

Background: $\mathrm{ABO}$ blood type incompatibility between donor and recipient represents a major hurdle given the high risk for antibody-mediated rejection (AMR). Incompatibility of $A B O$ blood type between donor and recipient is one of the major barriers to transplantation. The prognosis of $\mathrm{ABO}$ incompatible (ABOi) living donor liver transplant (LDLT) has improved dramatically since the introduction of rituximab. However, pre-transplant high isoagglutinin (IA) titers are considered to be major risk factor of AMR. Nevertheless, several protocols have been applied to overcome high antibody titer. Here, we report a successful ABOi LDLT in high pre-transplant IA titer recipient.

Case report: The recipient was a 55-year-old male who had alcoholic liver cirrhosis with blood type O+. He received ABOi LDLT from his son of blood type B+. His initial IA immunoglobulin G (IgG) titer was 1:1,024. Rituximab of 375 mg/BSA was given before 2 weeks of expected transplantation. However, the antibody titer did not decease, so we postponed the transplantation. He received 11 times of plasma pheresis. His last IA IgG titer was 1:128. The right liver of $802 \mathrm{~g}$ was donated and graft to recipient weight ratio was 1.20. Booster rituximab $200 \mathrm{mg}$ single dose was given at postoperation day (POD) 2. At POD 6, IA titer was raised to 1:64. We treated him with two times of plasma pheresis every other day. Subsequently bile has been well drained through external bile stent. We did not any more plasmapheresis even though IgG titer was over 1:32 after POD 13. Six months after the transplant, he has maintained stable liver function.

Conclusions: The most important factor for preventing AMR in recipients undergoing ABOi LDLT is the suppression of de novo antibodies. If the pre-transplant IA titer does not decease as low as the target, the protocols of well-combined with booster rituximab, high dose intravenous IG, and salvage plasmapheresis are considered to be able to overcome in ABOi LDLT.

Corresponding author: Dong Hoon Shin

E-mail: surgeonshin@naver.com

(C) The Korean Society for Transplantation

This is an Open Access article distributed under the terms of the Creative Commons Attribution Non-Commercial License (http://creativecommons.org/licenses/by-nc/4.0/) which permits unrestricted non-commercial use, distribution, and reproduction in any medium, provided the original work is properly cited. 